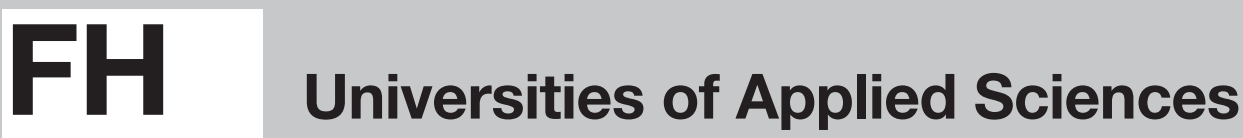

Fachhochschulen - Hautes Ecoles Spécialisées

\section{Keeping SARS-CoV-2 out: Vaccines, Filters, and Self-disinfecting Textiles}

Dieter Eibla, Martin Sievers ${ }^{a}$, Regine Eibla, Martin Bachmann $^{b}$, Senta Walton ${ }^{c}$, Nik Keel ${ }^{\mathrm{d}}$, Gilles Richnere, Stefan Stübingerf, Sven Hirsch ${ }^{9}$, Chahan Yeretziana, Sebastian E. W. Opitz ${ }^{\mathrm{a}}$, Trond Heldal ${ }^{\mathrm{h}}$, and Christian Adlhart ${ }^{\star a}$

${ }^{*}$ Correspondence: Prof. Dr. C. Adlharta, E-mail: christian.adlhart@zhaw.ch anstitute of Chemistry and Biotechnology, Zurich University of Applied Sciences ZHAW, Einsiedlerstrasse 31, CH-8820 Wädenswil, Switzerland; 'Department of Rheumatology, Immunology and Allergology, Inselspital, University of Bern, CH-3010 Bern, Switzerland; 'Saiba Biotech, Bahnhofstr. 13, CH-8808 Pfäffikon, Switzerland; dTB-Safety AG, Untere Grubenstrasse 3, CH-5070 Frick, Switzerland; 'Division of NBC-Protection, Spiez Laboratory, Austrasse, $\mathrm{CH}$ 3700 Spiez, Switzerland; 'Department of Biomedical Engineering, University of Basel, Gewerbestrasse 14, CH-4123 Allschwil, Switzerland; Institute for Applied Simulation, ZHAW; ' $O s m o t e x ~ A G$, Schützenstrasse 3, CH-8800 Thalwil, Switzerland

Abstract: When the SARS-CoV-2 pandemic started, ${ }^{[1]}$ science came to the immediate attention of the broad public. People and politicians were hanging on every word of medical doctors, virologists, molecular biologists, data scientists and many others in the hope of finding other protective measures than those used for centuries such as basic hygiene, distance, or quarantine. Here, at the Institute of Chemistry and Biotechnology at the Zurich University of Applied Sciences (ZHAW) we were also willing to provide scientific solutions to overcome the pandemic. Together with our partners from industry, we contributed to the development of a Swiss vaccine, are working on filters for active ventilated full protective suits and are developing tests to show the efficacy and safety of an active antiviral textile that allows controlled virus inactivation through an electrochemical reaction by applying a small current.

Keywords: Antiviral textiles - Pandemic $\cdot$ Personal protective equipment $\cdot$ SARS-CoV-2 $\cdot$ VLP-based vaccine

\section{Development of an Upstream Production Process for a VLP-based Human Vaccine against SARS-CoV-2}

The availability of an effective and safe vaccine to prevent SARS-CoV-2 provides a solution to overcome the current pandemic. The first two approved vaccines are BNT $162 \mathrm{~b} 2$ from BioNTech/Pfizer and mRNA-1273 from Moderna. More than 50 other candidates are in phase I, II or III clinical trials, according to ClinicalTrials.gov and the WHO. In addition to mRNA-based vaccines, the main candidates are inactivated virus, non-replicating viral vector, protein subunit and viruslike particle (VLP)-based vaccines. A promising approach for rapid and scalable SARS-CoV-2 VLP production is the expression of viral protein in Escherichia coli, which was investigated on behalf of Saiba Biotech. The two subtasks to be realized included production clone optimization and reproducible production of VLPs in stirred bioreactor systems with working volumes of $250 \mathrm{ml}$ and 301 .

\section{Protein Expression for Generation of a Vaccine Candidate against SARS-CoV-2 ${ }^{\dagger}$}

SARS-CoV-2 binds to the host receptor ACE2 via the receptor binding domain (RBD) of the spike glycoprotein. The RBD of SARS-CoV-2 has a length of 194 amino acids, is glycosylated and contains disulfide bonds. The RBD contains antigenic epitopes and can be used as a subunit vaccine candidate. ${ }^{[2]}$

Two gene cassettes each under the control of the T7 promoter integrated into a pETvector (Merck Millipore) for expression in $E$. coli were obtained from Saiba Biotech. The gene cassettes encode for two proteins able to assemble into VLPs. The two proteins are composed of a short peptide of RBD fused to the coat protein of the cucumber mosaic virus, containing a small fragment of the tetanus neurotoxin representing a universal T cell epitope, and of the structural capsid protein from the cucumber mosaic virus, respectively. VLPs derived from cucumber mosaic virus displaying RBD were immunogenic and generated antibodies in mice, which were able to block the binding of lentiviral particles pseudotyped with the spike glycoprotein to Human Embryonic Kidney cells expressing ACE2 at their surface. ${ }^{[3]}$ The two proteins used for production of VLPs were expressed in different $E$. coli strains. The following $E$. coli strains were used: BL21(DE3), BL21(DE3) transformed with the plasmid pGro7, ClearColi(DE3), C2566, Origami 2(DE3), OverExpress C41(DE3), Rosetta-gami 2(DE3), Tuner(DE3). The different strains used for expression under various growth conditions should minimize the formation of inclusion bodies and should enhance the formation of soluble protein product. The most soluble protein of $24.6 \mathrm{kDa}$ and $35.3 \mathrm{kDa}$ was produced in $E$. coli Tuner(DE3) (Merck Millipore, Sigma-Aldrich 70623) using sequence-optimized sequences and 2-YT broth for cultivation.

\section{Controlled and Scalable Upstream Production Process for SARS-CoV-2 VLPS}

The upstream production process consists of a growth phase and a production phase. The latter serves to express the target protein and is initiated by the addition of induction agents such as isopropyl $\beta$-D-1-thiogalactopyranoside (IPTG). Establishing scalable VLP production required screening studies and the design of an experiment method at $\mathrm{ml}$ scale to determine the most suitable culture media and process-specific parameters (optimal concentration of IPTG, induction time, growth and production temperature, initial cell or optical density, OD) to guarantee the desired quantity and solubility of the VLPs. For this purpose, three production clones were cultivated in the BioLector microbioreactor (high-throughput bioreactor system from $\mathrm{m} 2 \mathrm{p}$ labs) using flower-shaped well plates for microbial fermentation ( $1.5 \mathrm{ml}$ working volume). The production clones were provided by the research groups of Prof. Martin Bachmann and Prof. Martin Sievers. The accompanying process analysis included: recording of $\mathrm{OD}_{600}$ values (BioSpectrometer Kinetic 6136), measurement of selected substrates/metabolites (CedexBio V2.3.0, Roche Diagnostics), determination of VLP quantity by SDS-Page and ELISA, and the assessment of VLP quality by electron microscopy.

In terms of biomass production, as well as VLP quantity and VLP quality, the complex 2-YT medium (Sigma-Aldrich) and the chemically defined medium according to Riesenberg et al. ${ }^{[4]}$ 
showed the best results. Both media were used in subsequent shake flask studies (using 250 and $500 \mathrm{ml}$ baffled shake flasks and the KuhnerTOM online gas analyzer) and modified for fedbatch applications (feeding). The codon optimized clone (clone no. 3) was found to give the highest product concentrations in the soluble supernatant and was therefore selected for the bioreactor experiments in the ambr 250 modular from Sartorius (Fig. 1) and the BIOSTAT D-DCU D-30-3 (Sartorius). The ambr experiments indicated a strong dependence of VLP concentration on the length of the production phase and the feeding regime implemented in the production phase. Thus, to ensure high VLP concentrations, excess of carbohydrates was to be avoided. In the ambr 250 modular as well as in the BIOSTAT D-DCU D-303 , the highest VLP concentrations were obtained using 2-YT medium. Although the maximum $\mathrm{OD}_{600}$ values (261.2) and total VLP concentrations $\left(2.55 \mathrm{~g} \mathrm{l}^{-1}\right)$ of the ambr 250 bioreactor runs were not achieved, the two proof-of-concept cultivations realized in the BIOSTAT D-DCU D-30-3 demonstrated the scalability of the developed upstream processing method for SARS-CoV-2 VLP production.

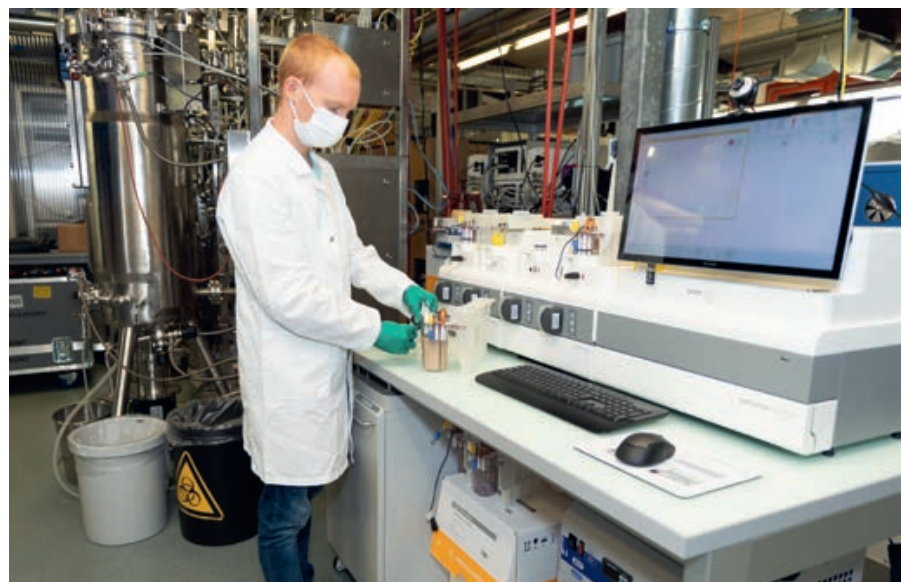

Fig. 1. ambr 250 modular used for the first scale-up step of the upstream processing of SARS-CoV-2 VLP production in the technology room of ZHAW's Center for Biochemical Engineering and Cell Cultivation Techniques (Photo: Patrick Cipriani)

\section{Filters for Full-protective Suits $\$$}

When the SARS-CoV-2 virus started spreading in Switzerland, a team at ZHAW was already working on 3D nanofiber-based aerosol filters. These filters are an integral part of an active ventilated protective suit (Fig. 2a). The project is funded by Innosuisse. ${ }^{[5]}$ Such protective suits were developed in response to previous pandemics, in particular the Ebola virus epidemic in 2014. The personal protective equipment should allow healthcare workers to treat patients while being fully protected themselves. A key factor is the simple handling of the protective gear; (i) since it needs to be donned correctly and (ii) during undressing contaminated outside surfaces must not be touched. Basic requests are high air circulation to limit the associated heat burden and an effective filter against pathogens with low-pressure drop. To fulfil these opposing requirements, filters are often made of several layers of different materials, some of which contain extremely thin nanofibers.

Aerosol filters are not simple sieves as several filtration mechanisms take place when submicron aerosols are trapped through fibrous media. These include inertial impaction, interception, diffusion, and electrostatic attraction. Inertial impaction, which happens when the particle cannot follow the air flow-field around the fibers, is mostly relevant for particles $>1 \mu \mathrm{m}$ because of their higher inertia. Smaller particles that are following the air streamline are caught through interception if they come within one particle radius of a fiber. Particles smaller than $0.2 \mu \mathrm{m}$ such as viruses exhibit significant Brownian motion and, thus, they no longer follow the air streamline. Indeed, they diffuse throughout the filter media until they touch a fiber surface. Diffusion capture becomes more effective with increasing filter surface and increasing retention time. At a given air flow, the retention time can be increased by extending the path length of the air stream through the filter media. In previous studies, we have investigated this effect by converting essentially flat 2D nanofiber mats (Fig. 2c) into extremely porous ultralight 3D nanofiber-based media, so-called nanofiber sponges or nanofiber aerogels (Fig. 2b).[6] Using the right processing conditions, we can tune the fiber density and pore size of these $3 \mathrm{D}$ filter media. ${ }^{[7]}$ With a thickness of $1 \mathrm{~cm}$ and a density of $8.7 \mathrm{mg} \mathrm{ml}^{-1}$, such materials achieved up to $99.998 \%$ filtration efficiency with an acceptable permeability of $57.7 \times 10^{-12} \mathrm{~m}^{2}{ }^{[8]}$ We can increase the permeability by increasing the pore size, but as with other filter media at the cost of filtration efficiency. While previous materials were made from biopolymers such as pullulan blended with poly(vinyl alcohol), filters in the current project are made from synthetic polymers such as polyacrylonitrile or polyamide. Filtration efficacy tests are performed at Spiez Laboratory (Fig. 2d).

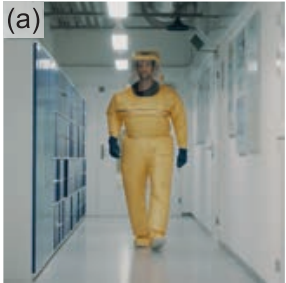

(d)

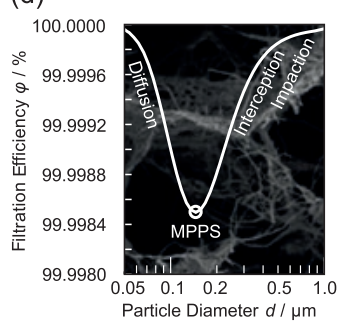

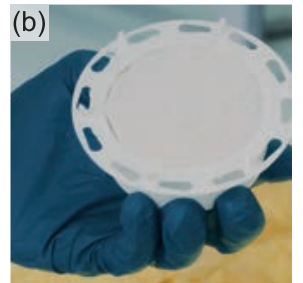

(e)
Fig. 2. Active ventilated protective suit (a) with integrated 3D nanofiber based filter (b); nanofiber production (c); fractional separation efficiency with most penetrating particle size (MPPS) and SEM image (d) and reconstructed $3 D$ structure based on $\mu C T$ scans $(e)$ of such filters.

To further direct the development of these next-generation nanofiber-based filter media, numerical simulations are performed. These simulations start from the actual filter geometries as derived from SEM images and $\mu \mathrm{CT}$ measurements (Fig. 2e). We are developing our own simulation environment, where the propagation of individual particles through the complex fibrous geometry is simulated by considering the stochastic Brownian dynamics and the forces governing particle-fiber interactions such as drag, friction, lift and adhesive forces. This approach gives more flexibility and control over the chemicophysical system parameters, although it requires significant simplifications. Simultaneously, we are also testing a voxelbased CFD commercial software to simulate more complex and realistic geometries. ${ }^{[9]}$ Currently, we are calibrating the physical model with real experimental data. In the near future, we will be able to predict the influence of changes of the $3 \mathrm{D}$ arrangement of the nanofibers within the filter media in terms of filtration 
efficiency and pressure drop. The ultimate challenge will be the industrialization of the filter production, but first estimates using current technology indicate manufacturing costs of $\mathrm{CHF}$ 10-20 per filter.

\section{Antiviral and Antibacterial Effects of a New Self-disinfecting Textile}

The use of mouth and nose covering textiles during the COVID-19 outbreak has become commonplace. Textiles act as mechanical filters and they may be equipped with passive antiviral coatings. Here we report on the development of textiles ${ }^{[10]}$ that actively and efficiently inactivate viruses and bacteria within minutes by application of a few volts. This involves research along (i) efficacy: develop and apply a protocol to measure the efficacy of microbial inactivation; (ii) safety: assess whether such products can be used without safety concerns; (iii) materials: selection, tuning and assembly of electrochemical textiles to optimize (i) and (ii).

\section{Efficacy}

Measurement of the efficacy of inactivation as a function of different parameters, applied to a range of viruses and bacteria is central to optimize and document the performance of antimicrobial textiles for various applications. In the initial assays, bacteriophage MS2 (DSM 13767), which is a singlestranded RNA coliphage, was used as indicator virus together with its host, the bacterium Escherichia coli (DSM 5695) as indicator bacterium. Bacteriophages are known to have stronger survival capability than most human pathogenic viruses and MS2 has been suggested to be used as a model organism for respiratory viruses in certification experiments to assess the potential of tissue membranes to inactivate viruses ${ }^{[11]}$ Requirements and test methods for antiviral textiles are described in the ISO 18184 standard for the determination of the antiviral activity of textile products. Next to the already established MS2 phage, also other viruses such as the feline calicivirus will be tested, in order to establish a standardized and fully validated test protocol for routine assessment of antiviral properties of textiles.

Table 1 documents the results for a series of efficacy measurement for textiles which combines passive inactivation via surface coating as well as active inactivation via application of a small voltage. The presently investigated textiles have demonstrated a faster and higher antiviral efficacy compared to passive antiviral textiles currently on the market. Further, the first commercial version (Osmotex Steriliser) is able to show $>95 \%$ virus inactivation in 15 minutes and $>99 \%$ in 2 hours, when applying the established protocol using MS2 phages developed at the ZHAW.
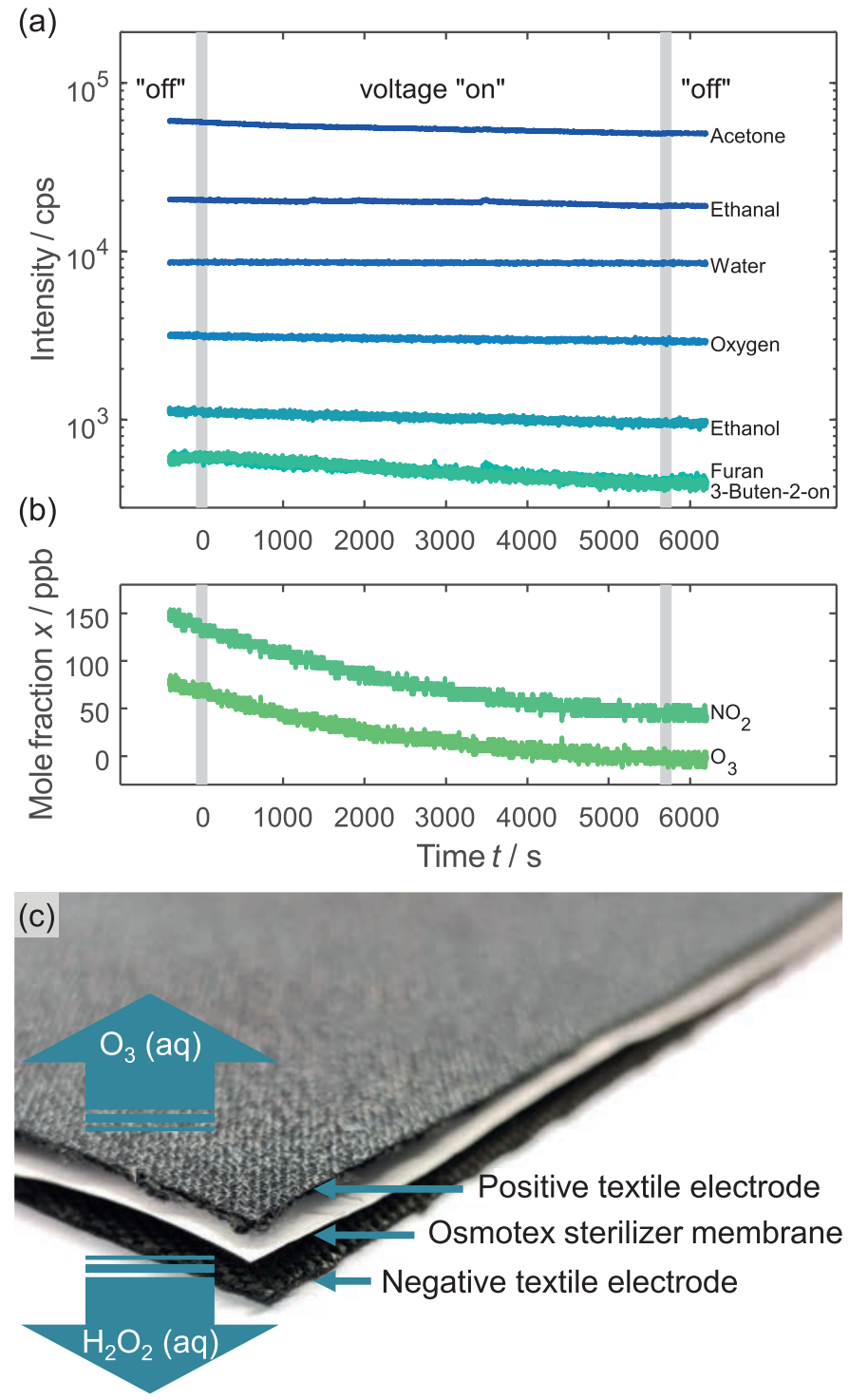

Fig. 3. The emissions for a large number of volatile organic compounds (VOCs) and hazardous gases from textiles were monitored in real time with (a) PTR-ToF-MS and electrochemical sensors (b) - here only a selection is shown. In this particular experiment, the pre-wetted textile was activated with $3 \mathrm{~V}$ direct current for 95 minutes in a closed box to document the safety of the voltage application and to demonstrate that no increase of VOCs or $\mathrm{NO}_{2}$ and $\mathrm{O}_{3}$ during voltage application was detected. (c) Structure and outline of the functionality of electrically controlled textiles for on-demand, self-disinfecting textiles for wearables and touch surfaces.

\begin{tabular}{|l|l|l|l|}
\hline Textile & $\begin{array}{l}\text { Time / } \\
\text { min }\end{array}$ & $\begin{array}{l}\text { Initial virus } \\
\text { concentration / } \\
\text { PFU ml-1 }\end{array}$ & $\begin{array}{l}\text { Efficacy / } \\
\text { \% inactivation }\end{array}$ \\
\hline $\begin{array}{l}\text { Passive antiviral mask 1 } \\
\text { (benchmarking 2, commercially available) }\end{array}$ & 15 & $10^{9}$ & $77 \pm 58$ \\
\hline $\begin{array}{l}\text { Passive antiviral mask 2 } \\
\text { (benchmarking 2, commercially available) }\end{array}$ & 15 & $10^{9}$ & $87 \pm 43$ \\
\hline $\begin{array}{l}\text { Osmotex Steriliser mask prototype (soon } \\
\text { commercially available) }\end{array}$ & 15 & $10^{7}$ & $\begin{array}{l}88 \pm 33 \\
95 \pm 4\end{array}$ \\
\hline $\begin{array}{l}\text { Osmotex Steriliser high performance materi- } \\
\text { al (not commercially available) }\end{array}$ & 15 & $10^{9}$ & $\begin{array}{l}>99.9997 \pm 0.0007 \text { (not a } \\
\text { single active virus could be } \\
\text { observed after test) }\end{array}$ \\
\hline Osmotex Steriliser mask prototype & 120 & $10^{7}$ & $99 \pm 1$ \\
\hline
\end{tabular}

Table 1. Results for different textiles that were tested with antiviral test protocols. These included the Osmotex Steriliser technology (first commercial textiles to be used in face masks) as well as passive sterilizing textiles, which are commercially available as masks, for benchmarking (measurement uncertainty: 95\% confidence interval). 


\section{Safety}

The targeted sterilization effect requires a controlled production of reactive oxygen species (ROS) such as $\mathrm{O}_{3}$ and $\mathrm{H}_{2} \mathrm{O}_{2}$, which is voltage- and time-dependent. While ROS have to be tuned to achieve the desired efficacy, they also need to be monitored and quantified. Furthermore, one has to monitor the formation and emission level of potentially harmful volatile organic compounds (VOCs) that might be generated during the application of a voltage. Accordingly, an analytical platform was developed to monitor the time- and spacedependent production and emission of ROC and VOCs from textiles. Knowledge of spatial emission patterns is crucial for improving the surface sterilization effect. Therefore, a sampling setup was established through coupling and adapting analytical technologies for the measurement of the volatile ROS and VOCs. Analytical techniques for VOC analysis include gas chromatography mass spectrometry for targeted identification and quantification of VOCs and proton-transfer-reaction timeof-flight mass spectrometry (PTR-ToF-MS) for time-resolved high-sensitivity untargeted analysis, ${ }^{[12]}$ as shown in Fig. 3a. To measure spatial emissions of harmful gases such as $\mathrm{NO}_{2}$ and $\mathrm{O}_{3}$, several electrochemical gas sensors have been used to detect potential gas emission in the ppb range, Fig. 3b. In addition, spectrometric methods are being deployed for quantitative analysis of hydrogen peroxide in solution.

\section{Material}

The basic design of first-generation textiles being developed consists of a three-layered design, see Fig. 3c; an insulating membrane between two conductive layers. An electrical voltage of 0.5 up to a few volts is applied via a rechargeable battery. In combination with humidity and air/oxygen, ROS are formed in the aqueous phase. Once these ROS come into contact with viruses on the textile, viruses (and bacteria) are destroyed within a few minutes.

The desired properties of the separator materials are ionic conductivity at low water content, facile wetting, elimination of the risk of direct contact between electrodes, low costs and flexibility. Textile electrodes should provide high conductivity, fine mesh, textile-like tactile feeling, moister withholding properties and the ability of coating. The contact layer properties depend on the properties of the electrode layers.

For now, a strong active virucidal and bactericidal effect was shown for the first generation of these new textiles, within minutes and with an efficiency better than 95\% (see Table 1). The self-disinfecting fabrics will be further optimized and they will be used to develop face masks and other commodities (bags, coats, seat covers, etc.) with on-demand sterilizing functionality, which could help to overcome the burden in daily life that is drastically restricted by the SARS-CoV-2 and potential future viral pandemics.

\section{Conclusion}

In the meantime, the development of SARS-CoV-2 vaccine in Switzerland was stopped, but the significance of bio manufacturing is still present. Full protective suites are not limited to SARSCoV-2 and smart sterilization may become an important tool in fighting daily health issues such as the flu, MRSA, and other hospital acquired infections.

\section{Acknowledgement}

We thank Nicola Barchi^ and Andreas Pfützner^ from Osmotex AG, Luca Huwyler ${ }^{\S}$ from Spiez Laboratory, Fabienne Arn ${ }^{\dagger}$, Gottfried Dasen^, Sebastian Eberle ${ }^{\ddagger}$, Lucie Filipovás ${ }^{\S \wedge}$, David Frasson ${ }^{\dagger}$, Géraldine Gubser $^{\ddagger}$, Ivana Kroslakova^, Nicola Lüdi ${ }^{\S}$, Rüdiger Maschke ${ }^{\ddagger}$, Jan Müller ${ }^{\ddagger}$, Sandro Roth ${ }^{\S}$, Cedric Schirmer ${ }^{\ddagger}$, Stefan Seidel ${ }^{\ddagger}$, Yannick Senn", Sandra Steiner ${ }^{\ddagger}$, Misha Teale ${ }^{\ddagger}$, Simone Ulzega ${ }^{\S}$, Tobias Wermelinger ${ }^{\dagger}$, and Dennis Wipfli^ from ZHAW for their assistance in realizing the experiments and evaluating the results.

Received: February 1, 2021

[1] a) D. M. Morens, P. Daszak, J. K. Taubenberger, N. Engl. J. Med. 2020, 382, 1293, https://doi.org/10.1056/NEJMp2002106; b) D Cucinotta, M. Vanelli, Acta Bio Med. Atenei Parmensis 2020, 91, 157, https://doi.org/10.23750/abm.v91i1.9397.

[2] a) L. Duan, Q. Zheng, H. Zhang, Y. Niu, Y. Lou, H. Wang, Front. Immunol. 2020, 11, https://doi.org/10.3389/fimmu.2020.576622; b) W.-H. Chen, U. Strych, P. J. Hotez, M. E. Bottazzi, Curr. Trop. Med. Rep. 2020, 7, 61, https://doi.org/10.1007/s40475-020-00201-6.

[3] L. Zha, H. Zhao, M. O. Mohsen, L. Hong, Y. Zhou, Z. Li, C. Yao, L. Guo, H. Chen, X. Liu, X. Chang, J. Zhang, D. Li, K. Wu, M. Vogel, M. F. Bachmann, J. Wang, bioRxiv 2020, 2020.05.06.079830, https://doi.org/10.1101/2020.05.06.079830.

[4] D. Riesenberg, V. Schulz, W. A. Knorre, H. D. Pohl, D. Korz, E. A. Sanders, A. Roß, W. D. Deckwer, J. Biotechnol. 1991, 20, 17 , https://doi.org/10.1016/0168-1656(91)90032-Q.

[5] C. Adlhart, M. Keller, 'Digital simulation for the tailored production of 3D nanofiber filters and their integration in a full protective suit for pandemic cases', Innosuisse, 35801.1 IP-ENG, 2019, https://www.aramis.admin.ch.

[6] a) F. Deuber, C. Adlhart, CHIMIA 2017, 71, 236, https://doi.org/10.2533/chimia.2017.236; b) P. Brodard, M. Dabros, R. Marti, E. Vanoli, M. Zinn, U. Frey, C. Adlhart, L. Kind, F. Koch, F. Burgio, J. Stenqvist, S. Saxer, U. Pieles, P. Shahgaldian, S. Wendeborn, CHIMIA 2019, 73, 645, https://doi.org/10.2533/chimia.2019.645.

[7] F. Deuber, S. Mousavi, M. Hofer, C. Adlhart, ChemistrySelect 2016, 1, 5595, https://doi.org/10.1002/slct.201601084.

[8] F. Deuber, S. Mousavi, L. Federer, M. Hofer, C. Adlhart, ACS Appl Mater Interfaces 2018, 10, 9069, https://doi.org/10.1021/acsami.8b00455.

[9] D. Hoch, M. Azimian, A. Baumann, J. Behringer, J. Niessner, 2020, 43, 2538, https://doi.org/10.1002/ceat.202000318.

[10] C. Yeretzian, T. Heldal, ' Electronically controlled sterilizing textile ', Innosuisse, 47326.1 IP-ENG, 2020, https://www.aramis.admin.ch.

[11] A. D. Coulliette, K. A. Perry, E. M. Fisher, J. R. Edwards, R. E. Shaffer, J. Noble-Wang, J. Int. Soc. Respir. Prot. 2014, 21, 14.

[12] F. Biasioli, C. Yeretzian, T. D. Märk, J. Dewulf, H. Van Langenhove, TrAC Trends Anal. Chem. 2011, 30, 1003, https://doi.org/10.1016/j.trac.2011.04.005. 